\title{
RADEMACHER SERIES WITH NONDIFFERENTIABLE SUMS
}

\author{
J. R. MCLAUGHLIN
}

A series of the form $\sum_{m=1}^{\infty} a_{m} r_{m}(t)$, where $\left\{a_{m}\right\}$ is a sequence of real numbers and $r_{m}(t)$ denotes the $m$ th Rademacher function, sign $\sin \left(2^{m} \pi t\right)$, is called a Rademacher series. Although a sufficient condition is known on the order of magnitude of the coefficients $\left\{a_{m}\right\}$ in order that $f(t) \equiv \sum a_{m} r_{m}(t)$ be differentiable almost everywhere, it remains to be determined if this condition is best possible. It is the purpose of this note to give conditions on the order of magnitude of the coefficients of a Rademacher series which are not sufficient in order to have $f(t)$ differentiable almost everywhere.

Previously, L. A. Balasov proved [1, p. 631] that $f(t)$ has a derivative at at least one point if and only if the limit of $\left\{2^{m} a_{m}\right\}$ exists and is finite. Balasov then showed that this condition on the coefficients is not sufficient for $f(t)$ to be differentiable almost everywhere $[1, \mathrm{p}$. 633]. More precisely, he exhibited a sequence $\left\{a_{m}\right\}$ for which

$$
\left|a_{m}\right|=O\left[2^{m} m^{1 / 2}\right]^{-1}
$$

and yet $f(t)$ was differentiable almost nowhere. In the opposite direction we have shown $\left[3\right.$, Theorem (3.1)] that if $\sum\left|a_{m}\right| 2^{m}$ converges, then $f(t)$ is of bounded variation. Hence, in particular, if

$$
\left|a_{m}\right|=O\left[2^{m} \cdot m \cdot \log m \cdot \log _{2} m \cdots\left(\log _{p} m\right)^{1+\epsilon}\right]^{-1}
$$

for some $\epsilon>0$ and some positive integer $p$, where $\log _{k} m$ denotes the $k$ th repeated or integrated logarithm of $m$ to the base 2 , then $f(t)$ is differentiable almost everywhere.

Thus naturally arises the question as to whether or not (2) is best possible, i.e. may we take $\epsilon=0$ for some positive integer $p$ and still obtain that $f(t)$ is differentiable almost everywhere. Although we cannot answer that question at present, we can decrease partially the gap between (1) and (2) by modifying Balasov's technique; and this is the purpose of this note.

We shall need the fact that if $t_{0} \in[0,1]$ has binary expansion $t_{0}$ $=. b(1) b(2) \cdots b(m) \cdots$ and is a dyadic irrational (i.e., not of the form $\left.p / 2^{k}\right)$, then

$$
r_{m}\left(t_{0}\right)=(-1)^{b(m)} .
$$

Received by the editors August 12, 1968. 
Also, we shall utilize the Borel-Cantelli lemma [2, p. 201] (cf. [1, p. $633]$ ) in the following form:

Lemma. Let $\left\{N_{j}\right\}$ be a sequence of nondecreasing positive integers, and let the sequence of integers $\left\{M_{j}\right\}$ be such that $M_{j} \geqq M_{j-1}+N_{j-1}$. Then in order that for almost every $t_{0} \in[0,1]$ the binary expansion $t_{0}$ $=. b(1) b(2) \cdots$ has the property that for infinitely many $j, b\left(M_{j}\right)$ is followed by $N_{j}$ zeroes, and for infinitely many $j_{1}, b\left(M_{j_{1}}\right)$ is followed by $N_{j_{1}}$ ones, it is necessary and sufficient that

$$
\sum_{i=1}^{\infty} 2^{-N_{i}}=\infty
$$

TheOREM. For every integer $p \geqq 2$ there exists a sequence $\left\{a_{m}\right\}$ satisfying

$$
\left|a_{m}\right|=O\left[2^{m} \cdot m \cdot \log _{2} m \cdot \log _{3} m \cdots \log _{p} m\right]^{-1}
$$

and yet $f(t) \equiv \sum a_{m} r_{m}(t)$ is differentiable almost nowhere.

Proof. Setting

$$
N_{j}=\left[\log \left(j \log j \cdot \log _{2} j \cdots \log _{p+1} j\right)\right]
$$

and

$$
M_{j}=\sum_{i=1}^{j-1} N_{i}
$$

we have that $\left\{N_{j}\right\}$ and $\left\{M_{j}\right\}$ satisfy the conditions of our lemma. In addition, notice that the sequence $\left\{M_{j}\right\}$ possesses the property

$$
M_{j} \leqq j N_{j} .
$$

Now consider the function $f(t)=\sum a_{m} r_{m}(t)$, where

$$
\begin{aligned}
a_{m} & =\left[2^{m} m \log _{2} m \cdot \log _{3} m \cdots \log _{p} m\right]^{-1}, \quad m=M_{j} \\
& =0, \quad m \neq M_{j}, \quad j=1,2, \cdots .
\end{aligned}
$$

This will be our desired function.

In order to prove that $f(t)$ is differentiable almost nowhere in $[0,1]$, we consider the set of dyadic irrationals $E \subset[0,1]$ whose elements have binary expansions with $N_{j}$ zeroes following $b\left(M_{j}\right)$ for infinitely many $j$. By our lemma the measure of $E$ is 1 , and therefore it will suffice to show that $f(t)$ has no derivative in $E$.

Assume now that $t_{0} \in E$, and that in the binary expansion of $t_{0}, N_{j_{k}}$ zeroes follow $b\left(M_{j_{k}}\right)$. Assuming for simplicity that $b\left(M_{j_{k}}\right)=1$, we choose $t_{k} \rightarrow t_{0}$ as $k \rightarrow \infty$ with the following binary expansion: 


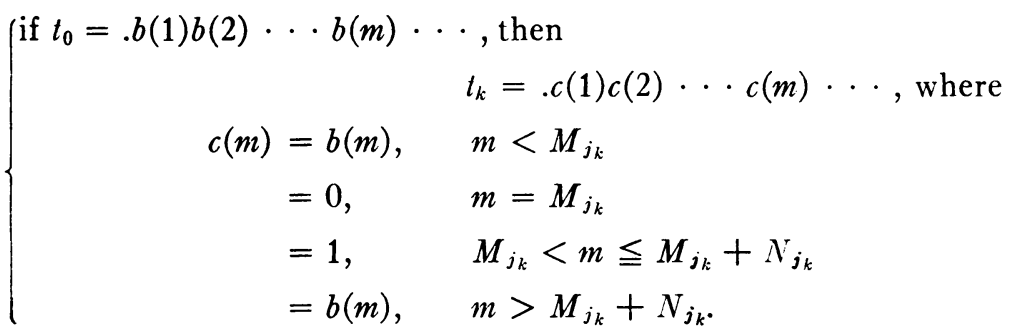

Thus, according to (3), (4), (5), and (6) we have:

$$
\begin{aligned}
\lim _{t_{k} \rightarrow t_{0}} \mid & {\left[f\left(t_{k}\right)-f\left(t_{0}\right)\right] /\left[t_{k}-t_{0}\right] \mid } \\
= & \lim _{t_{k} \rightarrow t_{0}}\left|\sum_{m=1}^{\infty} a_{m}\left[r_{m}\left(t_{k}\right)-r_{m}\left(t_{0}\right)\right] /\left[t_{k}-t_{0}\right]\right| \\
= & \lim _{k \rightarrow \infty} \mid 2 \cdot 2^{N_{j_{k}}}\left[M_{j_{k}} \log _{2} M_{j_{k}} \cdots \log _{p} M_{j_{k}}\right]^{-1} \\
& -\left[2\left(M_{j_{k}}+N_{j_{k}}\right) \log _{2}\left(M_{j_{k}}+N_{j_{k}}\right) \cdots \log _{p}\left(M_{j_{k}}+N_{j_{k}}\right)\right]^{-1} \mid \\
\geqq & \lim _{k \rightarrow \infty} 2^{N_{j_{k}}}\left[M_{j_{k}} \log _{2} M_{j_{k}} \cdots \log _{p} M_{j_{k}}\right]^{-1} \\
\geqq & 2^{-1} \lim _{k \rightarrow \infty}\left[j_{k} \log j_{k} \cdots \log _{p+1} j_{k}\right]\left[M_{j_{k}} \log _{2} M_{j_{k}} \cdots \log _{p} M_{j_{k}}\right]^{-1} \\
\geqq & 2^{-1} \lim _{k \rightarrow \infty}\left[j_{k} \log j_{k} \cdots \log _{p+1} j_{k}\right]\left[j_{k} N_{j_{k}} \log _{2} j_{k} N_{j_{k}} \cdots \log _{p} j_{k} N_{j_{k}}\right]^{-1} \\
\geqq & K \lim _{k \rightarrow \infty} \log _{p+1} j_{k}=\infty,
\end{aligned}
$$

where $K$ is some positive constant. Hence, $f^{\prime}\left(t_{0}\right)$ does not exist.

In the general case, where $b\left(M_{j_{k}}\right)$ is not always 1 , we let $b(N)$ be the nearest nonzero digit satisfying the condition $N \leqq M_{j_{k}}$ and choose $j$ such that $M_{j-1}<N \leqq M_{j}$. Then for $t_{k}$ we will take the point

$$
\begin{array}{rlrl}
t_{k} & =. c(1) c(2) & \cdots c(m) \cdots, \text { where } \\
c(m) & =b(m), & & m<N \\
& =0, & & m=N \\
& =1, & & N<m \leqq M_{j+1} \\
& =b(m), & & m>M_{j+1}=M_{j}+N_{j}
\end{array}
$$


and by means of analogous reasoning obtain the same result. This completes our proof.

\section{REFERENCES}

1. L. A. Balasov, Series with gaps, Izv. Akad. Nauk SSSR Ser. Mat. 29 (1965), 631-644; English transl., Amer. Math. Soc. Transl. (2) 77 (1968), 1-15.

2. P. R. Halmos, Measure theory, Van Nostrand, Princeton, N. J., 1950.

3. J. R. McLaughlin, Functions represented by Rademacher series, Pacific J. Math. 27 (1968), 373-378.

Pennsylvania State University 